International Journal of Pure and Applied Mathematics

Volume 98 No. 3 2015, 309-312

ISSN: 1311-8080 (printed version); ISSN: 1314-3395 (on-line version)

url: http://www.ijpam.eu

doi: http://dx.doi.org/10.12732/ijpam.v98i3.2

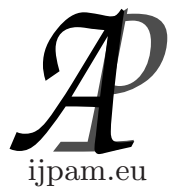

\title{
HYPER PATHS AND HYPER CYCLES
}

\author{
R. Dharmarajan ${ }^{1}$, K. Kannan ${ }^{2}$ \\ ${ }^{1,2}$ Department of Mathematics \\ SASTRA University \\ Thanjavur, Tamilnadu State, INDIA
}

\begin{abstract}
In graphs, paths are walks with no repeated vertex. A fortiori, paths cannot have any repeated edge. But in hypergraphs, hyperedges can repeat in vertex-to-vertex walks without causing repetition of any vertex. This is the crux of the idea of generalizing paths and cycles (from graphs to hypergraphs) presented in this short article.
\end{abstract}

AMS Subject Classification: 05C12, 05C38, 05C65

Key Words: hypergraph, hyperedge, adjacency, path, cycle

\section{Introduction}

A simple hypergraph [3] is an ordered couple $H=(V, E)$ where: (i) $V$ is a nonempty finite set and (ii) $E$ is a set of nonempty subsets of $V$ such that $\bigcup_{X \in E} X=V$. Each member of $V$ is a vertex; and each member of $E$ is a hyperedge (or, an edge). The hypergraph $H$ is Sperner if the members of $E$ have the property that none is a subset of another.

The cardinality [5] of a set $X$ is denoted by $|X|$. A hyperedge $X$ with $|X|=1$ is a loop. A hypergraph is loop-free if $|X|>1$ for each hyperedge $X$.

Received: April 26, 2014

(C) 2015 Academic Publications, Ltd.

${ }^{\S}$ Correspondence author url: www.acadpubl.eu 
Two distinct vertices $x$ and $y$ are adjacent if there is a hyperedge that contains both $x$ and $y$. A simple loop-free graph is a simple loop-free hypergraph with the additional stipulation that $|X|=2$ for each hyperedge $X$. In the context of graphs, hyperedges are usually called edges.

The contributions of this article are generalizations of paths and cycles (from graph theory) to hyper paths and hyper cycles, respectively. Much of the motivation for this research work comes from: (i) varying definitions of acyclic hypergraphs (section 5 of [2]), and (ii) path formation in hypergraphs by sequences of hyperedges (section 3 of [2]). Perforce, more stipulations have been incorporated into the latter (see section 2 that follows) so that it can be successfully particularized to graphs (section 3). All the hypergraphs in the coming discussions are assumed Sperner and loop-free unless there are explicit and unambiguous indications to the contrary.

\section{Definitions}

A hyper path in $H=(V, E)$ between two distinct vertices $x_{1}$ and $x_{k}$ is a sequence $x_{1}, A_{1}, \ldots, x_{k-1}, A_{k-1}, x_{k}$ with the following properties: (i) $k$ is a positive integer $\geq 2$; (ii) $x_{1}, \ldots, x_{k}$ are distinct vertices; (iii) $A_{1}, \ldots A_{k-1}$ are hyperedges (not necessarily distinct); and (iv) $x_{j}, x_{j+1} \in A_{j}$ for $j=1$ through $k-1$. This hyper path is also denoted by: (i) $P\left(x_{1}, \ldots, x_{k}\right)$; or (ii) $P=x_{1}, \ldots, x_{k}$; or (iii) $P\left(x_{1}, x_{k}\right)$; or, simply, (iv) $P$. The set $r(P)=\left\{x_{1}, \ldots, x_{k}\right\}$ is the range of $\mathrm{P}$. The positive integer $|r(P)|-1$ is the length of $\mathrm{P}$, and is denoted by $\|P\|$. The points $x_{1}$ and $x_{k}$ are the end points (or, terminal points) of $\mathrm{P}$. If the hyperedges $A_{1}, \ldots, A_{k-1}$ are also distinct, then the hyper path $P$ is a path between $x_{1}$ and $x_{k}$. $H$ is connected if there is a hyperpath between $x$ and $y$ whenever $x$ and $y$ are distinct vertices in $H$.

A hyper cycle in $H=(V, E)$ at a vertex $x_{1}$ is a sequence $x_{1}, A_{1}, \ldots, x_{k-1}, A_{k-1}$, $x_{k}, A_{k}, x_{1}$ with the following properties: (i) $k$ is a positive integer $\geq 3$; (ii) $x_{1}, A_{1}, \ldots, x_{k-1}, A_{k-1}, x_{k}$ is a hyper path between $x_{1}$ and $x_{k}$; (iii) at least one among $A_{1}$ through $A_{k-1}$ is distinct from $A_{k}$; (iv) $x_{j}, x_{j+1} \in A_{j}$ for $j=1$ through $k-1$; and (v) $x_{k}, x_{1} \in A_{k}$. This hyper cycle is also denoted by: (i) $C\left(x_{1}, \ldots, x_{k}, x_{1}\right)$; or (ii) $C=x_{1}, \ldots, x_{k}, x_{1}$; or (iii) $C\left(x_{1}\right)$; or, simply, (iv) $C$. The set $r(C)=\left\{x_{1}, \ldots, x_{k}\right\}$ is the range of $\mathrm{C}$. The positive integer $|r(C)|$ is the length of $\mathrm{C}$, and is denoted by $\|C\|$. If $A_{1}, \ldots, A_{k}$ are also distinct, then the hyper cycle $C$ is a cycle at $x_{1} . H$ is hyper acyclic if it has no hyper cycles.

Clearly: (i) a hyper cycle $C$ begins and ends at $y$ for each vertex $y \in r(C)$; and 
(ii) an acyclic graph is a hyper acyclic hypergraph with $|X|=2$ for each hyperedge $X$.

The distance between two vertices $x$ and $y$ in $H=(V, E)$ is denoted by $d(x, y)$, and is defined as follows: $d(x, y)=0$ if $x=y ; d(x, y)=\min \{\|P\|$ : $P$ is a hyper path between $x$ and $y\}$ if $x \neq y$. Evidently: (i) $d(x, y)$ defines a metric [1] on $V$; (ii) $d(x, y)=1$ if $x \neq y$ and $x$ is adjacent to $y$; and (iii) in a simple loop-free graph, $d(x, y)$ is the number of edges in any shortest path between $x$ and $y$.

\section{Main Results}

Proposition 3.1. If $P$ is a hyper path between $x_{1}$ and $x_{k}$ then $P$ contains a path between $x_{1}$ and $x_{k}$.

Proof. Induction is used on $k$. For $k=2$, any hyper path $x_{1}, A_{1}, x_{2}$ is clearly a path. Assume the result for $k=r$. Then let $P$ be a given hyper path between $x_{1}$ and $x_{r+1}$, written $x_{1}, A_{1}, \ldots, x_{r}, A_{r}, x_{r+1}$. Then $x_{1}, A_{1}, \ldots, A_{r-1}, x_{r}$ is a hyper path (call it $\mathrm{Q}$ ) between $x_{1}$ and $x_{r}$. By induction hypothesis, $\mathrm{Q}$ contains a path between $x_{1}$ and $x_{r}$, say: $x_{1}, B_{1}, \ldots, B_{t}, x_{r}$ where the $B$ 's, like the $x$ 's, are distinct. At this point:

(i) if $A_{r}=$ some $B_{s}(1 \leq s \leq t)$, then $x_{1}, B_{1}, \ldots, B_{s}, x_{r+1}$ is a path between $x_{1}$ and $x_{r+1}$; or,

(ii) if $A_{r}$ equals none of $B_{1}$ through $B_{t}$, then $x_{1}, B_{1}, \ldots, B_{t}, x_{r}, A_{r}, x_{r+1}$ is a path between $x_{1}$ and $x_{r+1}$.

Proposition 3.2. In a simple, loop-free graph $G=(V, E)$, each hyper path is a path.

Proof. In $G$, if an edge repeats in a hyper path, then the vertices in that edge must repeat in the hyper path. But this is contradictory to the definition of a hyper path.

Example 3.3. A hyper cycle need not contain a cycle. Consider $H=$ $(V, E)$ where $V=\{1,2,3,4,5,6,7\}, E=\left\{A_{1}, A_{2}, A_{3}\right\}$ with $A_{1}=\{1,2,3\}, A_{2}=$ $\{2,3,4,5\}$ and $A_{3}=\{3,5,6,7\}$. Then $C\left(1, A_{1}, 2, A_{2}, 3, A_{1}, 1\right)$ is a hypercycle, but $C$ contains no cycles - because omission of any hyperedge from $C$ leaves not more than two vertices standing, thus ruling out any chance of a cycle.

Proposition 3.4. In a simple, loop-free graph $G=(V, E)$, each hyper cycle is a cycle. 
The proof is similar to that of 3.2 .

\section{Summing Up}

Generalizing results from graph theory is one of the main purposes of the theory of hypergraphs [4]. For a generalization of this kind to be successful, its particularization from hypergraphs to graphs has to follow unambiguously when the additional stipulation for graphs (that $|X|=2$ for each hyperedge $X$ ) is factored in. The generalizations of paths and cycles (to hyper paths and hyper cycles, respectively) are successful in this regard, corroborated by 3.2 and 3.4 . What seems promising, at this point, is that more ideas from graph theory (degree and neighborhood of a vertex, to mention two) could be generalized, besides, if at all, identifying graph-theoretical ideas that might not be amenable to generalization to hypergraphs.

\section{Acknowledgments}

The authors express their thanks to (1) Professor R. Sethuraman, Vice Chancellor, SASTRA University and (2) D. Ramachandran, Director, Abel Mathematics Foundation, Palghat (Kerala State, India) for their unstinted encouragement and support.

\section{References}

[1] T.M. Apostol, Mathematical Analysis, Addison-Wesley, Reading, 1974.

[2] C. Beeri, R. Fagin, D. Maier and M. Yannakakis, On the desirability of acyclic database schemes, Journal of the Association for Computing Machinery, 30 (1983), 479-513.

[3] C. Berge, Hypergraphs - Combinatorics of finite sets, North-Holland, Amsterdam, 1989.

[4] C. Berge, Graphs and Hypergraphs, North-Holland, Amsterdam, 1973.

[5] R. R. Stoll, Set Theory and Logic, Dover, New York, 1963. 